\title{
A novel in treatment of cancer anal canal
}

\author{
Emad M Salah, MD; Tarek Gobran, MD; Doaa O Refat, $M D$
}

\author{
Department of Surgery, Zagazig University, Egypt.
}

\begin{abstract}
Background: Posterior sagittal approach offers a direct exposure to the rectum, a better definition of the striated muscle complex, and a more objective way to reconstruct the arrangement between the neorectum and the muscle complex. Similar approaches have been used successfully for rectal and sigmoid resection for Hirschsprung's disease and rectal cancer.

The aim of this study was to assess the long term oncologic and functional results of a new technique of posterior sagittal approach for excision of anal canal and rectum and relocating the sigmoid colon into the tract of longitudinal striated muscle fibers in treatment of superficial cancer of anal canal.

Method: 13 patients presenting with anal cancer were submit to posterior sagittal incision for resection of anal canal, rectum and mesorectum and relocation of the sigmoid colon within the anal sphincter.

Results: This study included 8 males (61.5\%) and 5 females (38.5\%), their age ranged from 58 to 73 years (mean age 64.3 years). Clinical examination and history showed bleeding in 6 patients (46.2\%), straining during bowel motion in 5 patients $(38.5 \%)$ and itching and change in bowel habits in 2 patients (15.4\%). Trans-rectal ultrasound of 5 patients showed that the anal cancer was limit to the anal canal and the mass was 2 to $4 \mathrm{~cm}$. (mean $2.9 \mathrm{~cm}$.) and in 8 patients the mass was located at the anorectal junction, mostly carcinoma in the distal rectum infiltrating the anal canal, the size of the mass 3 to $5 \mathrm{~cm}$. (mean $4.2 \mathrm{~cm}$.). Histological examination: 8 patients $(61.5 \%)$ diagnosed as adenocarcinoma were 4 males (30.8\% and 4 females $(30.4 \%)$ and 5 patients (38.5\%) diagnosed as squamous cell carcinoma were 4 males (28.28\%) and one female (7.7\%). The mean operating time was 285 minutes ranging from 165 to 290 minutes. The longer operating time was usually at the first few cases at the beginning of the study. The mean length of resected part of the anal canal and rectum was $14.6 \mathrm{~cm}$. ranging from 13 to 18 $\mathrm{cm}$. The neorectum was well vascularized and its wall was kept uninjured during the procedure. There were no intra-operative or early postoperative complications, no patients had wound infection during the first 3 weeks, 4 patients had 6-11 bowel motions per day whereas the others had only 3 movements daily. Subsequently the frequency of bowel motions became normal in all patients within 4 months with 1 to 3 bowel motions per day. Follow-up for all patients must be at regular interval every 3 months for per-rectal examination and 6 months for transrectal ultrasound to detect any local recurrence. Two patients only had local recurrence at 34 and 30 months after surgery and were treated by abdominoperineal resection and permanent colostomy.

Conclusion: The posterior sagittal approach seems to be a reliable method for resection of anal canal and rectum in treatment of superficial cancer of anal canal uT1 and uT2 provided that regular follow-up is adopted at 3 to 6-month intervals.

Key word: Posterior sagittal, incision, anal canal cancer.
\end{abstract}

\section{Introduction:}

The posterior sagittal approach allows complete anatomic exposure of the perineum and pelvis. Moreover, it reduces the risk of damaging important structures because the incision is in the midline. Therefore many surgeons have used these approaches in diseases other than anorectal malformations, including intestinal dysganglinosis, trauma, parasacral mass and rectal duplication. ${ }^{1}$ 
Posterior midsagittal approach was used for the first time in 1980 to treat anorectal malformations. This approach provides wide exposure of the lower rectum and pelvic floor, through a midline posterior incision, to determine the limits of the sphincteric mechanism and place the rectum within its limits. ${ }^{3}$

Pena et al $(1993)^{4}$ evaluated the effects of the posterior sagittal approach on rectal function and fecal continence (experimental study). The study comprised four groups of four animals. Animals were evaluated clinically and manometrically pre and postoperatively. It was found that the perirectal dissection without posterior sagittal incision provoked the more sever changes in the bowel control and manometric study. Posterior sagittal approach with or without rectotomy provoked minimal or no changes in bowel functions and manometric study. So the posterior sagittal approach does not interfere with the function of the sphencteric mechanism.

Anal cancer is an often curable disease, the 3 major prognostic factors are site (anal canal versus perianal skin), size (primary tumor less than 2 centimeters in size have a better prognosis) and differentiation (well differentiated tumors are more favorable than poorly differentiated tumors). 5,6

The aim of this study is to assess the long term oncologic and functional results of a new technique of posterior sagittal approach for excision of anal canal and rectum and relocating the sigmoid colon into the tract of longitudinal striated muscle fibers in treatment of superficial cancer of anal canal.

\section{Patients and methods:}

Between January 2006 to January 2008, 13 patients presenting with anal cancer , 8 males and 5 females, the age range from 58 to 73 years (mean age 64.3 years) were submited to posterior sagittal incision for resection of the anal canal, rectum and mesorectum and relocation of the sigmoid colon within the anal sphincter.

Clinical examination and history showed bleeding in 6 patients (46.2\%), straining during bowel movement in 5 patients $(38.5 \%)$ and itching and change in bowel habits in 2 patients $(15.4 \%)$.

\section{Pre-operative investigations:}

All patients were submitted to per-rectal examination to assess the extent of the mass and mobility as only small masses from 2 to $5 \mathrm{~cm}$, mobile and not infiltrating the perianal skin were selected in this study.

\section{Transrectal ultrasound:}

For all patients to determine the depth of the tumors as we selected the patients with only uT1 and uT2. The transrectal sonographic classification of the tumor invasion into bowel wall (uT) corresponds to pathologic classification (pT) of the TMN system, in which uT1 represents mucosal or submucosal disease, uT2 is disease involving the hypoechoic muscularis propria, uT3 is disease extension into the anal sphincter and uT4 is disease into adjacent organs or sidewall structures.

\section{Computed tomography (CT):}

To the pelvis and abdomen to detect any pelvic lymph node or other organ affection.

Other pre-operative investigations as cardiological examination, liver and renal functions assessment were done.

\section{Perioperative Management:}

The colon was prepared for 3 days preoperatively with saline enemas, oral manitol and oral metronidazol 500mg T.D.S. and cefotaxime $1 \mathrm{gm} / 12$ hour. The patients were fed with a low residue diet. Twelve hours before the surgery, the patients fasted, and also for the first 5 days after surgery, and intravenous fluid, cefotaxime and metronidazole were given.

\section{Surgical procedure:}

After induction of general anesthesia and endotracheal intubation, the patient was positioned in a prone jack-knife position. The rectum was then packed with gauze to prevent contamination of the wound during rectal mobilization. A midline skin incision was made from the level of the midsacrum to the anus. The coccyx, the levator muscle, and the striated muscle complex were divided in the midline, make the incision in the center of the muscle the levator or the muscle complex was pushed up and divided down to the anal dimple along its longitudinal fibers. The fascia of Waldeyer was opened, and the rectal wall was exposed. Multiple fine sutures were tagged at the mucocutaneous junction for traction. 
A circumferencial incision was made around the anus, and the plane of cleavage between the rectal wall and the surrounding tissue was developed. The rectum was mobilized by dissection close to the adventitia rectalis, which could be identified by traction on the rectum and distinguished from the surrounding muscle complex. This dissection was performed all the way up to the supralevator space, and, eventually, the peritoneal reflection was reached and opened anteriorly and laterally. A loop of rectum gradually was mobilized by dividing of vessels and bands posteriorly and laterally. By traction on the rectum, the mesenteric vessels and bands could be exposed easily, ligated, and divided under direct vision. Provided that the rectal mesentery was freed, the sigmoid could be pulled easily through out the incision by moderate traction. The mesentery of the sigmoid colon was divided and freed without tension up to the proposed anastomotic line. Attention should be taken to preserve the colonic vascular arcades, which approach the colonic wall from either side. The entire rectum and anal canal were resected, and a new anus was reconstructed by relocating the sigmoid colon into the tract of the longitudinal striated muscle fibers and attaching to the muscle complex anteriorly and posteriorly. Because the diameter of the colon usually is larger than the width of the longitudinal muscle tract, the posterior wall of the colon was folded inward longitudinally rather than excised to fit the size of the muscle tract. The wound was closed in layers, and a new anal opening was made according to the limit of the longitudinal muscle tract. Suction drains were placed and removed 48 hours postoperative. Anal dilatation was started fifteen days after the operation. All patients underwent regular follow-up in our outpatient department at regular 3- to 6-months intervals.

The parameters described by Pena were used for clinical evaluation of the bowel function.

1. Voluntary bowel movement is defined as the act of feeling the urge to use the toilet and holding the bowel movement until the patient reaches the bathroom.

2. Soiling is defined as the involuntary leaking of small amount of stool. This sign is quantified as grade 1 when the soiling occurs occasionally in minimal amounts, and the patient has no social problem. Grade 2 refers to soiling that occurs every day but does not cause any social problems. Grade 3 refers to soiling that is constant and represents a social problem to the patient.

3. Constipation is defined as the incapacity to empty the rectum spontaneously every day. It is quantified as grade 1 when the constipation is manageable by changes in diet, grade 2 when the patient requires laxatives, and grade 3 when the patient requires enemas.

\section{Results:}

The patients in this study included 8 males $(61.5 \%)$ and 5 females $(38.5 \%)$. Their age ranged from 58 to 73 years (mean age 64.3 years)

Clinical examination and history showed bleeding in 6 patients (46.2\%), straining during bowel movement in 5 patients (38.5\%), itching and change in bowel habits in 2 patients (15.4\%).

Trans-rectal ultrasound of 5 patients showed that the anal cancer was limited to the anal canal and the mass 2 to $4 \mathrm{~cm}$. (mean $2.9 \mathrm{~cm}$.) and in the other 8 patients the mass was located at the anorectal junction, mostly carcinoma in the distal rectum infiltrating the anal canal, the size of the mass was 3 to $5 \mathrm{~cm}$. (mean 4.2 $\mathrm{cm}$.).

CT. scan showed no enlarged mesorectal lymph node.

Histological examination: 8 patients $(61.5 \%)$ diagnosed as adenocarcinoma were 4 males $(30.8 \%)$ and 4 females $(30.8 \%)$ and 5 patients (38.5\%) diagnosed as squamous cell carcinoma were 4 males $(28.28 \%)$ and one females $(7.7 \%)$.

The mean operating time was 285 minutes ranging from 165 to 290 minutes. The longer operating time was usually at the first few cases at the beginning of the study.

The mean length of resected part of the anal canal and rectum was $14.6 \mathrm{~cm}$. ranging from 13 to $18 \mathrm{~cm}$. The neorectum was well vascularized and its wall was kept uninjured during the procedure.

All patients started postoperative bowel function within the first 24 hours and oral feeding was resumed on the sixth day. 
There were no intra-operative or early postoperative complications, no patients had wound infection during the first 3 weeks, 4 patients had 6-11 bowel movements per day whereas the others had only 3 to 4 movements daily, subsequently the frequency of bowel movements became normal in all patients within 4 months with 1 to 3 bowel movement per day. Daily dilatation was carried out for 3 months to avoid stenosis.

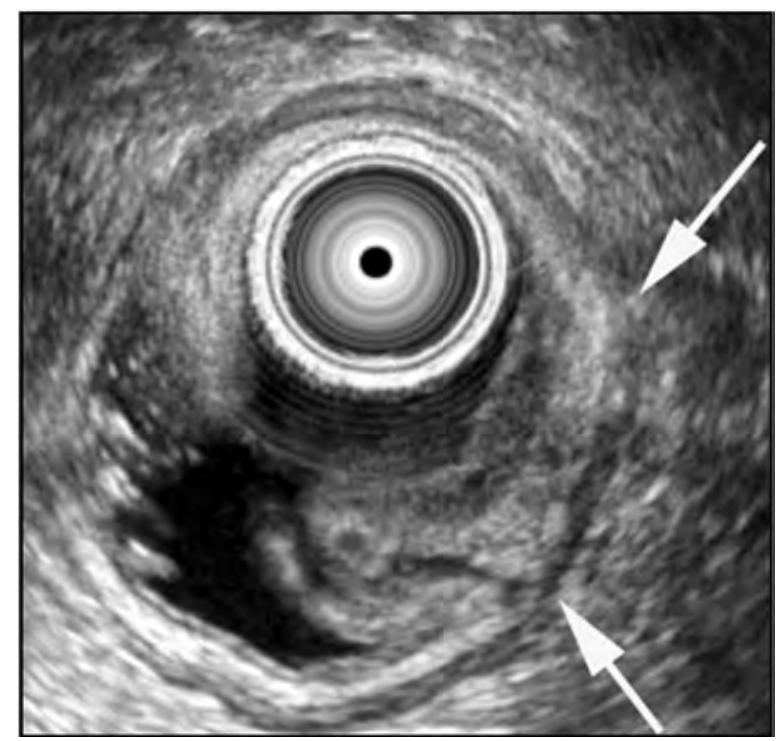

Figure (1): Transrectal ultrasound showed small hypoechoic mass confined to mucosal and submucosal layers with (uT1).

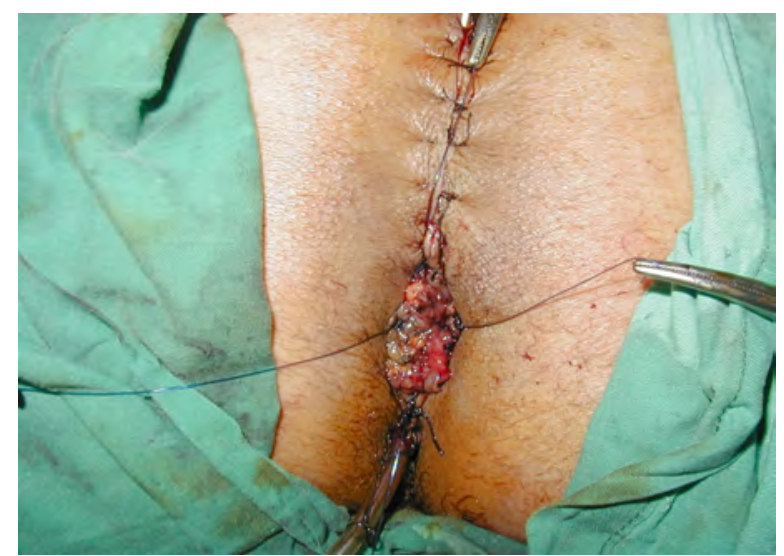

Figure (3): Pose operative closure of the wound with suction drain and new anus formation.

\section{Discussion:}

Local excision and adjuvant chemoradiation therapy of anal cancer had a high rate of recurrence as the resection margin, usually 1 to $2 \mathrm{~cm}$, may be a positive resection margin. ${ }^{7}$

Following initial radiotherapy or chemoradiotherapy for the treatment of anal
Follow-up for all patients must be at regular interval every 3 months for per-rectal examination and 6 months for transrectal ultrasound to detect any local recurrence. 2 patients only had local recurrence at 30 and 34 months after surgery and were treated by abdominoperineal resection and permanent colostomy. The mean follow up period was 28 months ranging from 11 to 45 months.

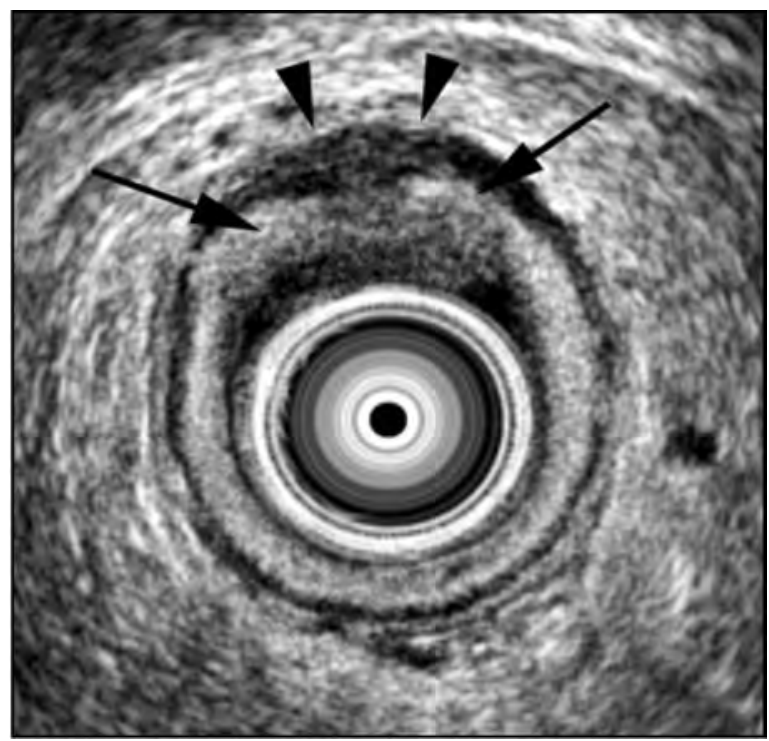

Figure (2): Transrectal ultrasound showed hypoechoic mass invading muscularis properia (uT2) with clear perirectal tissue.

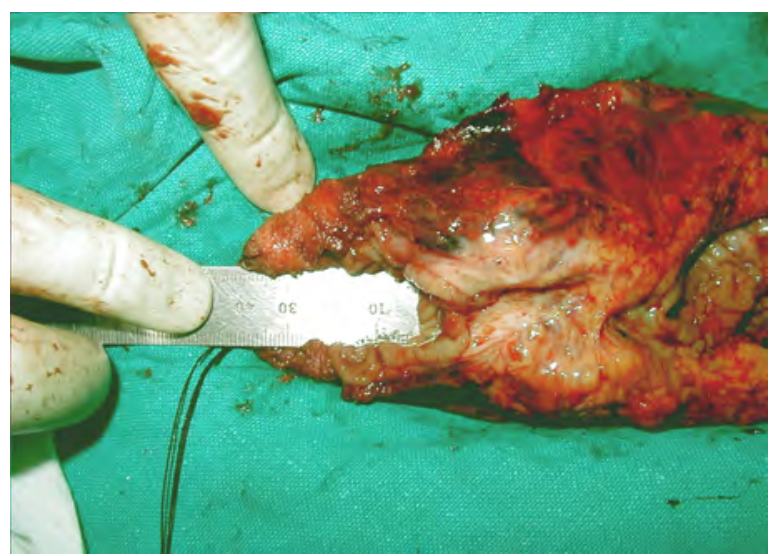

Figure (4): Post operative spacemen showing tumor $4 \mathrm{~cm}$ within the anal canal.

cancer, patients who present with either persistent or locally recurrent disease are treated by abdominoperineal resection which is more extensive and does not preserve the anal sphincter. As a result the patient needs a permanent colostomy. ${ }^{8}$ 
Intersphincteric resection for very low rectal cancer infiltrating the anal canal, a distal resection margin of $1 \mathrm{~cm}$ can be obtained by partial intersphincteric resection. This method is associated with many complications as local recurrence in $17.4 \%$, an anastomotic leakage in $8.8 \%$ and anovaginal fistula in $2.1 \% .9,10$

The posterior sagittal approach offers a direct exposure to the rectum, a better definition of the striated muscle complex, and a more objective way to reconstruct the arrangement between the neorectum and the muscle complex. Similar approaches have been used successfully for rectal and sigmoid resection for Hirschsprung's disease and rectal cancer. We believe that wound infection after posterior sagittal approach mainly results from tension and poor vascularization at the anorectal anastomotic line. In the current approach, the sigmoid colon is released fully, its mesenteric vascular arcades are preserved, and the neorectum is located in the muscle complex without any tension. This is the reason that no wound infection occured in our series even though colostomy has not been performed. ${ }^{11}$

One potential hazard with this technique is uncontrolled bleeding from the mesenteric vessels when the mesocolon is being divided, so extreme care should be taken to obtain hemostasis by ligation of the mesenteric vessels and bands of the colon. Another potential hazard is necrosis of the neorectum, which could be avoided by preserving the straight colonic arteries, which go laterally to the bowel. ${ }^{11}$

Conclusion: The posterior sagittal approach seems to be a reliable method for resection of anal canal and rectum in treatment of superficial cancer of anal canal uT1 and uT2 provided that follow-up is adopted regularly at intervals from 3 to 6-month.

\section{References:}

1- Pini Prato A, Martucciello G, Torre M, Jasonni V: Feasibility of posterior sagittal approaches in patients without anorectal malformations. Pediatr Surg Int 2004; 20(20): 762-767.

2- Pean A: The posterior sagittal approach : Implications adult colorectal surgery. Dis Colon Rectum 1994; 37(1): 1-11.
3- Keramidas DC, Soutis M, Koulpoulos K, Moschov D: The posterior sagittal approach for the surgical excision of intrapelvic malignant tumours. Eur J pediatric Surg 2003; 13(4): 245-248.

4- Pena A, Amroch D, Baeza C, Csury L, Rodriguez G: The effects of posterior sagittal approach on rectal function (experimental study). J Pediatr Surg 1993; 28(6): 773-778.

5- Martenson JA, Lipsitz SR, Lefkopoulou M: Results of combined modality therapy for patients with anal cancer: An Eastern Cooperative Oncology Group study. Cancer 1995; 76(10): 1731-1736, 6- Fuchshuber PR, Rodriguez-Bigas M, Weber $\mathrm{T}$ : Anal canal and perianal epidermoid cancers. Journal of the American College of Surgeons 1997; 185(5): 494-505.

7- Lee J, Corman M: Recurrence of anal adenocarcinoma after local excision and adjuvant chemoradiation therapy: Report of a case and review of the literature. $J$ Gastrointest Surg 2009 ;13: 150-154.

8- Mariani P, Ghanneme A, De la Rochefordière A, Girodet J, Falcou MC and Salmon RJ: Abdominoperineal resection for anal cancer. Dis Colon Rectum 2008; 51(10): 1495-1501.

9- Chamlou R, Parc Y, Simon T, Bennis M, Dehni N, Parc R, Tiret E. Long term results of intersphincteric resection for low rectal cancer. Ann Surg 2007; 246(6): 916-921.

10-Akasu T, Takawa M, Yamamoto S, Ishiguro S, Yamaguchi T, Fujita S, Moriya Y, Nakanishi Y: Intersphincteric resection for very low rectal adenocarcinoma: Univariate and multivariate analyses of risk factors for recurrence. Ann Surg Oncol 2008; 15(10): 2668-2676.

11-Long LI, Wang YX, Zhang J Z: Posterior sagittal approach: Megasigmoid resection and anal reconstruction for severe constipation and fecal incontinence after anoplasty. Journal of Pediatric Surgery 2000; (35) 7: 1058-1062. 